\title{
Enhancing Mechanical Stability of Nano-Structured Anti-Reflection Coatings
}

\author{
Behnam Kheyraddini Mousavi1, Tito Busani², Mani Hossein Zadeh², Steven Roy Julien Brueck ${ }^{2}$ \\ ${ }^{1}$ Department of Electromechanical Engineering, Northern New Mexico College, Espanola, USA \\ ${ }^{2}$ Department of Electrical Engineering, University of New Mexico, Albuquerque, USA \\ Email: behnam.mousavi@nnmc.edu,busanit@unm.edu,mhz@unm.edu,sbrueck@unm.edu
}

How to cite this paper: Mousavi, B.K., Busani, T., Zadeh, M.H. and Brueck, S.R.J. (2020) Enhancing Mechanical Stability of Nano-Structured Anti-Reflection Coatings. Journal of Applied Mathematics and Physics, 8, 247-258.

https://doi.org/10.4236/jamp.2020.82020

Received: January 7, 2020

Accepted: February 3, 2020

Published: February 6, 2020

Copyright (C) 2020 by author(s) and Scientific Research Publishing Inc. This work is licensed under the Creative Commons Attribution International License (CC BY 4.0).

http://creativecommons.org/licenses/by/4.0/

\begin{abstract}
Periodic Nanostructured anti-reflection coatings (NALs) are a promising option for enhancing transmission of coherent light without inducing scattering. We've found that reducing the height of NALs below a critical value to enhance mechanical stability can highly reduce the transmission efficiency. Here, using Rigorous Couples Wave Analysis (RCWA), we find the minimum height for over $99 \%$ transmission and effect of height on transmission bandwidth. Then, during a one-step plasma etching, two samples with different heights have been generated and their efficiency is evaluated using RCWA.
\end{abstract}

\section{Keywords}

Maximum Transmission, Mechanical Stability, Reflection, Laser, Nano-Structure

\section{Introduction}

Conventional ARCs coatings are based on destructive interference [1] [2] [3] at a certain wavelength, enhancing optical transmission at that specific wavelength. Using multiple layers for broadening the transmission spectrum results in considerable residual stresses [4], lowers the tolerance and life of the multi layer AR coating; specially for high power laser applications [5]. Nanotechnology has introduced a broad span of new options for overcoming obstacles in science [6] and engineering [7] [8] [9] from sensing [10]-[14] to mechanical characteristics of materials [15] [16] which was not before possible. Nanostructured anti-reflection coatings (NALs) are the new generation of anti-reflection coatings designed to deal with previous problems.

These nanostructured materials work based on a fill factor concept [1] (fill factor relates the volume occupied with a dielectric to an effective refractive in- 
dex of the dielectric). Such coatings are widely used for increasing light transmission to solar cells [17] [18], and light extraction from LED's [19] [20] and lasers [21] [22]. Sub-wavelength NAL structures are usually carved out of substrate and thus, are made of the same material as the underlying medium. NALS are used for different applications. For example, these structures can be used for increasing transmission in infrared detectors [23]. By using NALs on materials transparent to IR, the detection capability of infrared cameras considerably has improved [24] [25]. For solar cells and LEDs, the goal is to enhance scattering with or without diffraction of light [17] [18] [26]. In fact, for such application, the important parameters are the peak transmission and the bandwidth.

Moreover, for laser applications, these nanostructures should be made in subwavelength periodic fashion for reducing scattering [22] [24] [27]. Silicon is transparent across a portion of near and mid-IR [28] but its high refractive index causes significant reflection at the silicon/air interface. Nanostructuring the surfaces of silicon can be used to improve transmission at the silicon/air interface in IR applications. As previously mentioned, one of the main applications of NALs is enhancing transmission of light through optical elements used in high-powerlaser applications [29] [30]. In such applications, it is also crucial to avoid scattering. Therefore, the randomly distributed NALs are replaced by ordered ones [24].

So far, considerable research has been done to fabricate NALs for enhancing transmission of IR [17] [23] [27] [31]. In most of these works, the height of nanostructure was optimized for boosting transmission without regard to mechanical stability and ruggedness. We will investigate the effect of height on the transmitted wavefront. We have fabricated an efficient profile for maximizing the transmission of light, while keeping the height of the nanostructures as low as possible. Shorter SiNPs are less prone to mechanical damage.

As the height of SiNPs decreases, the transmission significantly reduces. While researchers have fabricated tall silicon nanostructures for suppressing reflection [22] [27], we have fabricated much shorter silicon nanostructures with over 99\% transmission. The transmission capability of the NAL structure has been evaluated using RCWA for different heights. It's shown that NALs designed for different wavelengths (with different heights), yield the same $99.1 \%$ transmission when their height results in a $\pi$ phase shift imposed on the transmitted light beam. It's seen that doubling the height of the nanopillars significantly widens the bandwidth lowering the dependence of the nanostructures on pitch. This is important if one wants the NALs to improve the transmission across a wider bandwidth. In such a broadband design, one wants to design the broadband nanostructures with a small pitch to avoid diffraction for lower wavelengths [7] [32].

In this paper, we evaluate the effect of height on transmission peak efficiency and bandwidth. After finding out the effect of height using RCWA method for a previously designed NAL [32], we proceed with fabricating NALs with two different heights. As the first step of fabrication, we use Interference lithography for 
patterning the silicon surface. Afterwards, the etching for generating tapered profiles is done using $\mathrm{CF}_{4}$ and $\mathrm{O}_{2}$ plasma. This is a single step process without the complexity of changing the plasma condition during the etch [33]. We have shown the resulting NALs using Scanning Electron Microscope (SEM). It's seen that using the lithography approach, the fill factor reduces due to empty spaces filled with air at the bottom ( Fill-Factor $=\frac{n_{\mathrm{Si}} \times A_{\mathrm{Si}}+n_{\text {Air }} \times A_{\text {Air }}}{2}$, where A stands for cross-section occupied at each elevation of the NAL). Enhancing the fill factor of the NALs at the base is a common problem for lithographically generated NALs [7] [27]. Clearly, at the base, there is an empty space between the NALs due to nature of the Lithography. The effectiveness of the fabricated nanostructures is evaluated using RCWA. Taller NALs result in much higher transmission. It's found that despite the empty spaces between the NALs, our NALs can result in a transmission above $92 \%$.

\section{Increasing Height to Wavelength Ratio}

The nanostructures profile studied in our previous work [32] generated by a logarithmic approach, had a height of $\frac{\lambda}{2 n_{\text {eff }}}$ which would result in a phase-shift of $\pi$ in the light as it travels through the nanostructure. For simplicity, we will refer to this height as $h_{\pi}\left(\sum_{i=1}^{N} \frac{\lambda}{2 n_{i} \cdot N}\right)$. This height is different for different profiles. The $h_{\pi}$ is actually half of the value commonly used in other reports [24] [27]. A NAL structure designed to generate a peak transmission for $4.72 \mu \mathrm{m}$ wavelength using logarithmic approach [32], is shown in Figure 1. Our simulation results indicate that increasing the nanostructure height further than $h_{\pi}$ doesn't result in any significant increase in transmission at the peak wavelength, however the bandwidth will improve significantly as shown in Figure 2.

Effect of height is more significant for high refractive index materials. That is, as the refractive index increases, a more gradual shift of the effective refractive index is needed and higher NALs must be applied for increasing the transmission. While examining effect of height for our silicon NALs, we want to find the minimum possible height for maximal transmission of light (to avoid wave-front diffraction).

Figure 3(a) \& Figure 3(b) show how transmission changes as a function of nanostructure height. Each of the nanostructures of Figure 3(a) is designed for a different $\lambda$ value. The selected pitch for each $\lambda$ value falls on the Pitch $=0.27 * \lambda$ line. This guarantees that the profile used will yield maximum transmission. As can be seen the transmission increases as the nanostructure height increases. The transmissions reach almost $100 \%$ as the height reaches $h_{\pi}$. As shown in Figure 3(b), all curves of Figure 3(a) overlap when the nanostructure-heights are normalized with respect to their corresponding $h_{\pi}$. Thus Figure 3 can be used to study the effect of nanostructure-height on transmission through NAL for all the wavelengths of interest. 


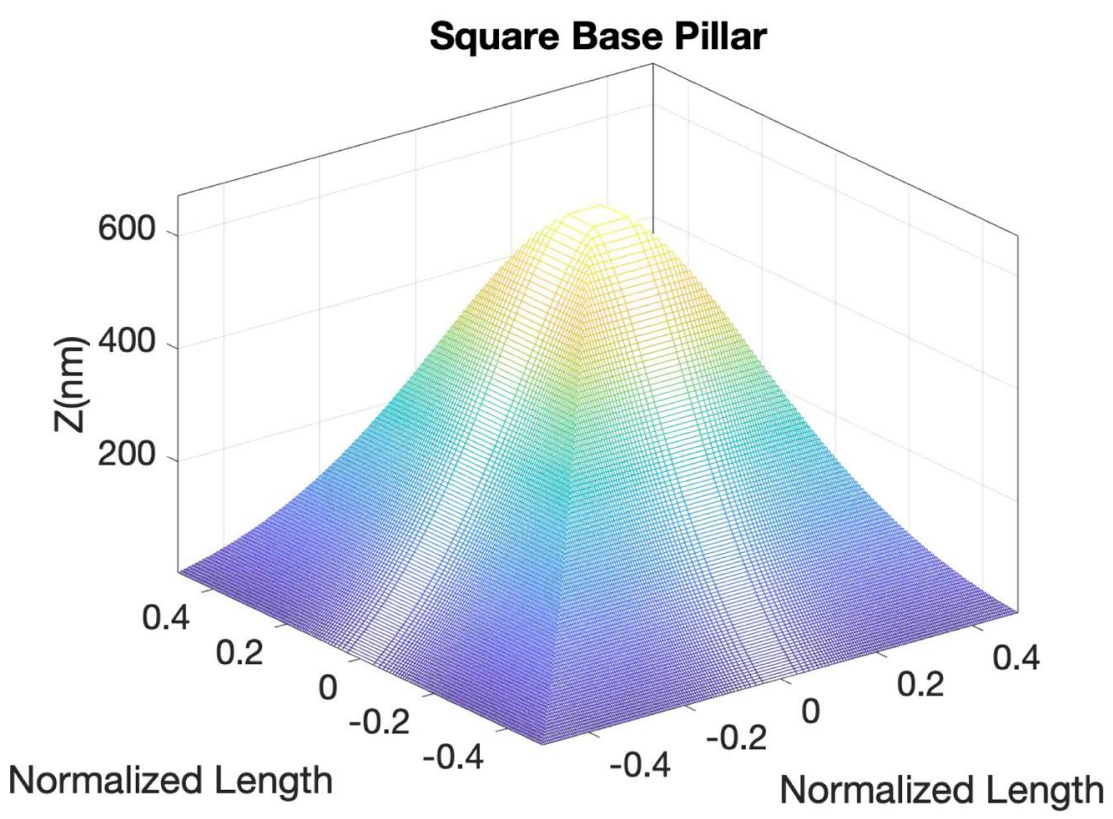

Figure 1. Profile of a NAL structure designed for $4.72 \mu \mathrm{m}$ using logarithmic approach [32].

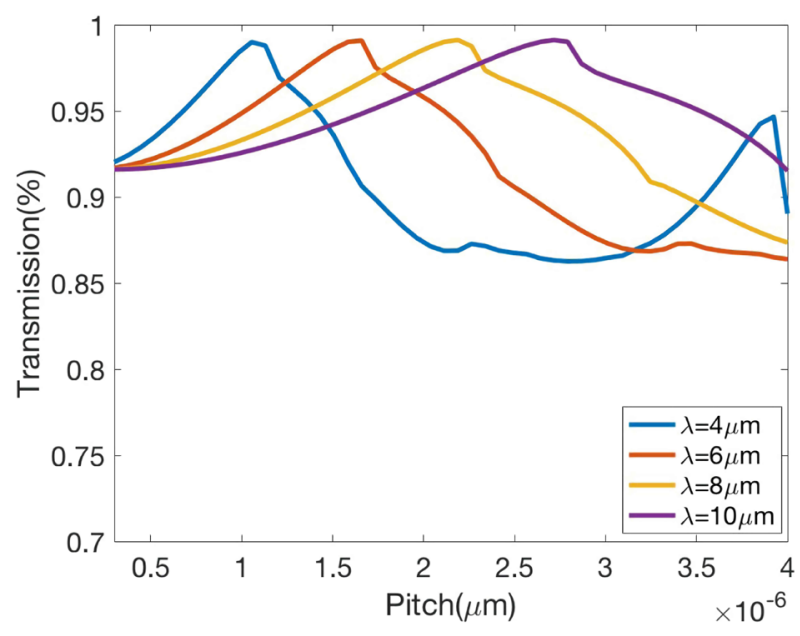

(a)

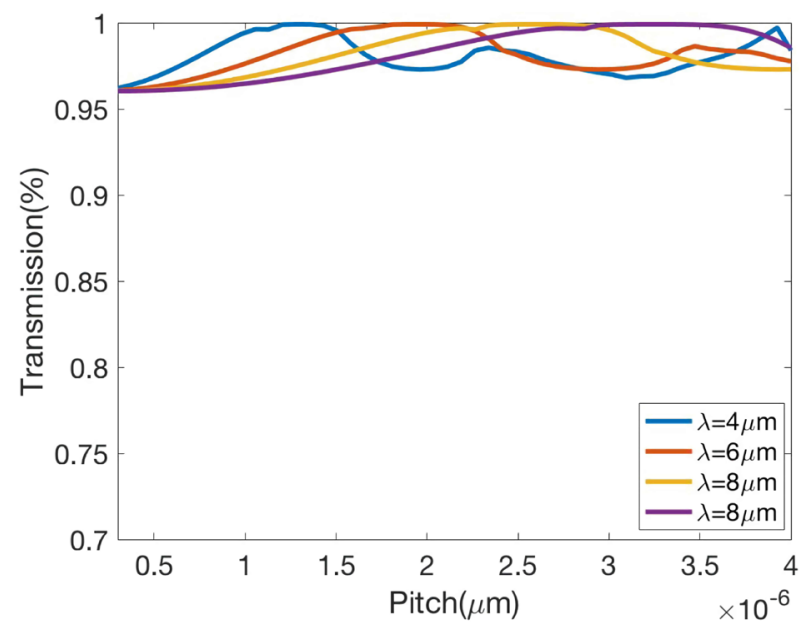

(b)

Figure 2. Comparison of the transmission bandwidth between two sets of nanostructures with heights of (a) $h_{\pi}$ and (b) $2 h_{\pi}$.

The results obtained here indicate that NALs with a thickness of $h_{\pi}$ provide maximum transmission while keeping the nanostructure heights at minimum. The wave-front passes without diffraction when the pitch for the logarithmic calculated profile is small enough ( Pitch $\leq \frac{\lambda_{\text {inc }}}{2 n_{\text {sub }}}$ ).

The results of this section indicate that $h_{\pi}$ is the optimal height for our nanostructures and in order to obtain maximum transmission at a target wavelength, the pitch should be set as using the Pitch $=0.27 * \lambda$ relationship. This pitch is related to our previously calculated profile [32]. 


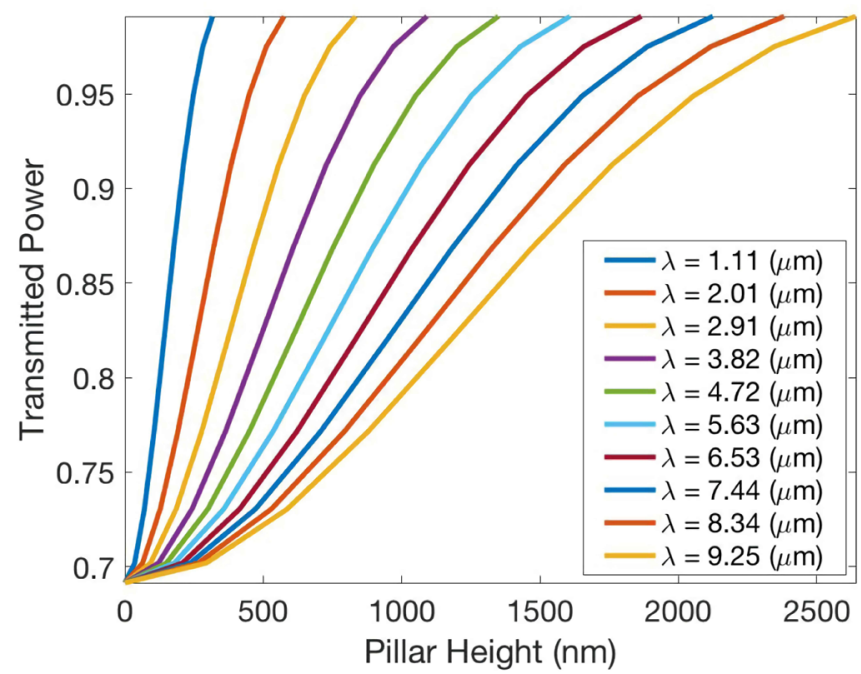

(a)

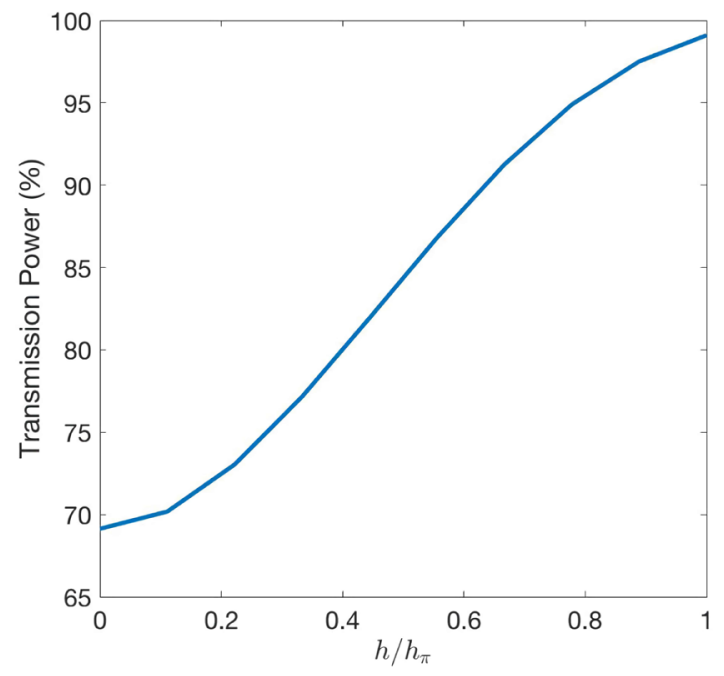

(b)

Figure 3. The transmission versus pillar height for different wavelengths. The total phase shift is $\pi$ where $T=99.1 \%$ (a) Transmission versus NAL height. Pitch $=0.27 * \lambda$ [32] (b) All transmission curves overlap when the nanostructure-heights are normalized with respect to the $h_{\pi}$ for each design.

\section{Fabrication Method}

In this section, we describe a process to fabricate tapered NALs in a single step without altering plasma condition or composition during the etching process. As discussed in previous section, as the height of the nanopillar decreases, the transmission decreases and its bandwidth narrows. This demands careful adjustment of pitch and profile for maximizing the transmission for the desired frequency. The fabrication process consists of patterning NR-7 negative photo-resist (over $70 \mathrm{~nm}$ Icon-7 ARC) by interferometric lithography (IL). An example of nanoholes generated by this approach is shown in Figure 4. As a first step of fabrication, the silicon wafers were cleaned by RCA I and RCA II. A thin $70 \mathrm{~nm}$ thin layer of Icon7 (ARC) coating was spin-coated on silicon wafer and baked at $200^{\circ} \mathrm{C}$. Afterwards a $500 \mathrm{~nm}$ thick NR7 photo-resist was coated over the ARC layer and backed at $110^{\circ} \mathrm{C}$ for $90 \mathrm{~s}$. After exposure, the photo-resist was baked again at $110^{\circ} \mathrm{C}$ for 1 minute and developed for $90 \mathrm{~s}$ (using $\mathrm{MF}_{321}^{\mathrm{TM}} / \mathrm{H}_{2} \mathrm{O}=3 / 1$ ) and then dried with a nitrogen gun. The next step is exposing the silicon on the developed areas by ashing the Arc layer using an oxygen plasma (Figure 4). Then, using E-beam deposition, the sample was coated with $\mathrm{Cr}$ (or Ni). After lift-off the mask is ready. The IL setup used for this purpose is shown in Figure 5.

A $355 \mathrm{~nm}$ wavelength, 3ns frequency tripled ND YAG laser is used for patterning the negative resist. The interference setup is shown in Figure 5, where the laser beam passes a lens and is collimated using a concave mirror. Half of the beam is directly incident on the sample while the rest of beam, first hits the mirror and gets reflected on the resist. This generates parallel line patterns on the photo-resist. Now, the sample is rotated $90^{\circ}$ and exposed to the IL laser again. 


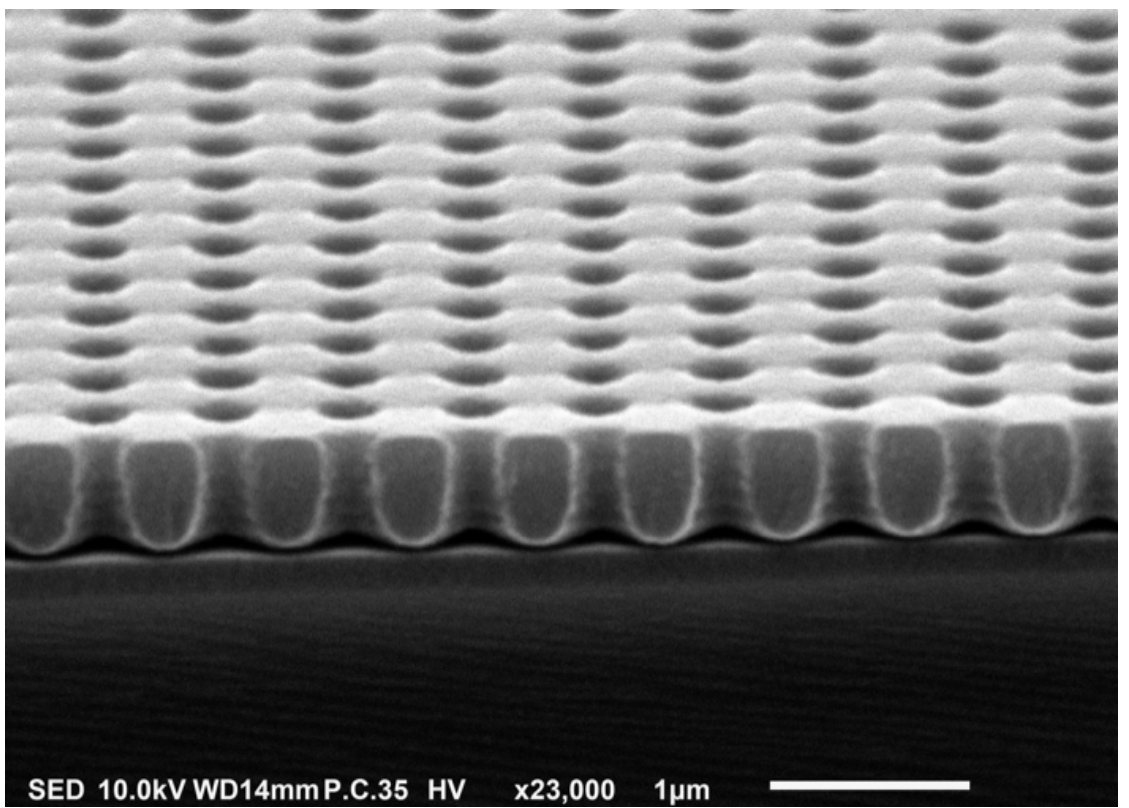

Figure 4. 2D photo-resist patterned created by the IL setup and $355 \mathrm{~nm}$ UV laser beam creating the low-pitch periodic nano-patterns on the photo-resist.

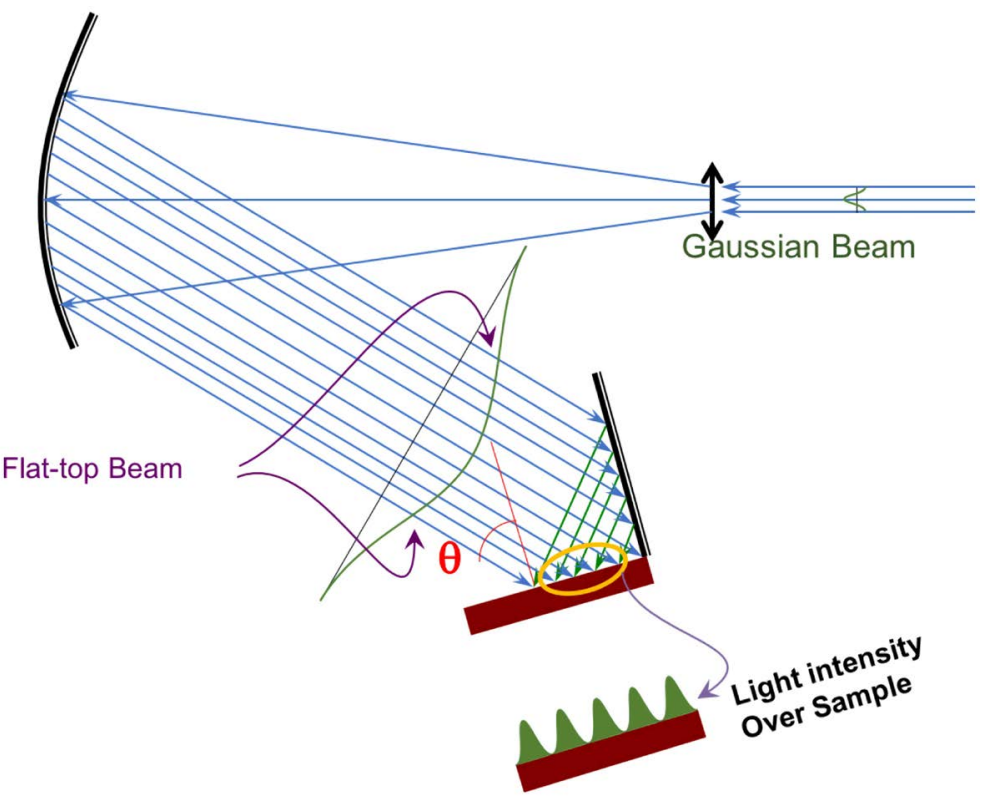

Figure 5. The IL setup showing the generation of the periodic nano-patterns on the photo-resist through interference.

The 2D pattern is now ready for being developed by developer solution. After developing the pattern (Figure 4) the masking metal will be deposited on the photo-resist and lifted off resulting in a periodic 2D pattern of metal dots. The metal mask preserves the silicon underneath it while the silicon elsewhere is etched by reactive ion etch process (RIE). The resulting straight NALs as seen in Figure 6 if only $\mathrm{CF}_{4}$ are used for etching.

Figure 7 illustrates the method to fabricate tapered NALs in a single step 


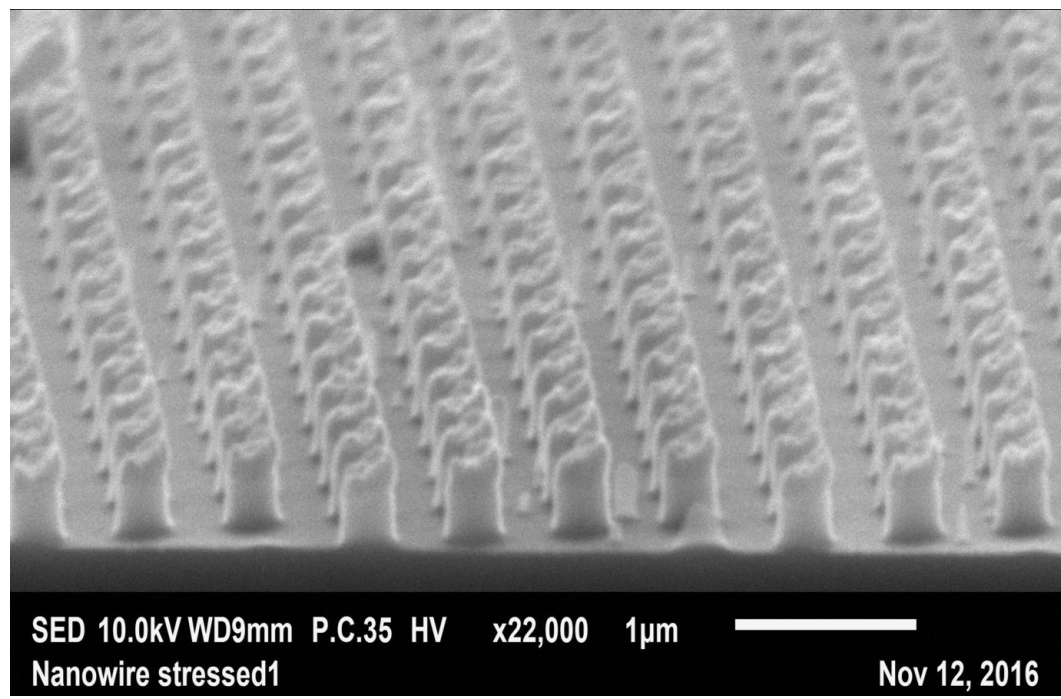

Figure 6. Metal cap on top of the straight NALs prevents the silicon under it from being etched by plasma. Absence of Oxygen has resulted in a vertical etch.

\section{RF Voltage}

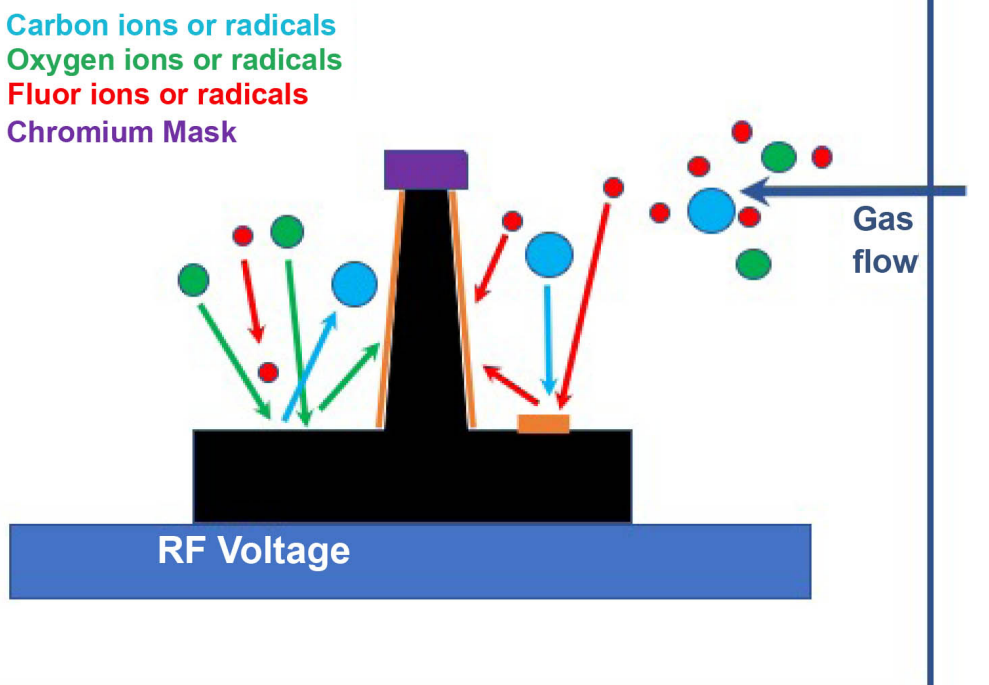

Figure 7. The ions or radicals of Carbon, Oxygen, and Fluor are shown in blue, green, and red. The teflone generated by carbon plasma, protects the Silicon from rather low energy fluor reactants but gets removed easily by oxygen.

without altering plasma condition or composition during the etching process. First Molecules containing Carbon, Oxygen, and Fluor enter the plasma chamber. The two big shown blue pads induce a very high voltage ionizing the molecules or generating radicals of the atoms. Carbon plasma bonds with silicon generating a teflon like long chain molecule. The long chain molecule protects Silicon from being etched by Fluor ions or radicals. The atoms of the plasma have the highest speed in vertical direction. Therefore, Oxygen can effectively remove 
the teflon on surface of substrate (leaving them exposed to Fluor), while it shallowly etches the teflon on the sidewalls of the NAL. By adjusting the amount of oxygen in chamber, one can decide the verticallity or slope of the side walls of the nanostructure. Low amount of Oxygen generates rather vertical sidewalls while higher amounts of Oxygen results in slanted side-walls. For etching the vertical pillars, we used 70 Watts, $\mathrm{CF}_{4}$ plasma (15SCCM flow) under $20 \mathrm{mToor}$ pressure and $0 \mathrm{SCCM} \mathrm{O}_{2}$ flow for 25 minutes. For etching short tapered NALs, we have used 50 Watts, $\mathrm{CF}_{4}$ plasma (15SCCM flow) under 20 mToor pressure and 2SCCM oxygen flow for 25 minutes. If we increase the power further, we risk to completely under etch the top of silicon nanopillar. Figure 8(a) shows short tapered NALs. The tapered profile is a result of $\mathrm{O}_{2}$ etching the side wall teflon [7] which usually form during etching silicon by $\mathrm{CF}_{4}$. For etching taller pillars with tapered sidewalls, we have used 120 Watts, $\mathrm{CF}_{4}$ plasma (15SCCM flow) under $20 \mathrm{mToor}$ pressure and 1SCCM oxygen flow for 25 minutes. While the reduction in Oxygen flow reduces the risk of excessive side etching, the higher power also increases the verticallity (compared to highly tapered NALs of Figure 8(a)), alongside etching depth at a fixed span of time.

Figure 8(b) shows the resulting tall-tapered NALs, which is a common problem for generating NALs using interferometric Lithography [7]. The transmission of the two short and tall nanopillars at the same pitch with different heights is shown in Figure 9. Note that due to fabrication limitations it's not possible to create the sharp tip seen in the taller NALs in shorter versions. However, due to the small cross-sections, the tips don't play a major role in increasing the transmission. The bottom side of the NAL plays a more critical role in amount of effective index. $n_{\text {eff }}=3.5 \times A_{\text {si-Cross-Section }}+1 \times\left(1-A_{\text {i-Cross-Section }}\right)$. Therefore $n_{\text {eff-Short-NAL }}=2.05$ and $n_{\text {eff-Tall-NAL }}=1.92$. If in our simulations we enter the same effective index for short NALs, its efficiency is still much less than the taller NALs.

We have emphasized the effect of height of nanopillars on bandwidth while showing that if designed for a single wavelength laser, the height of NALs can be taken quite short. This significantly enhances the mechanical stability. Profiles with tapered sidewalls are fabricated. However, our fabricated nanostructures

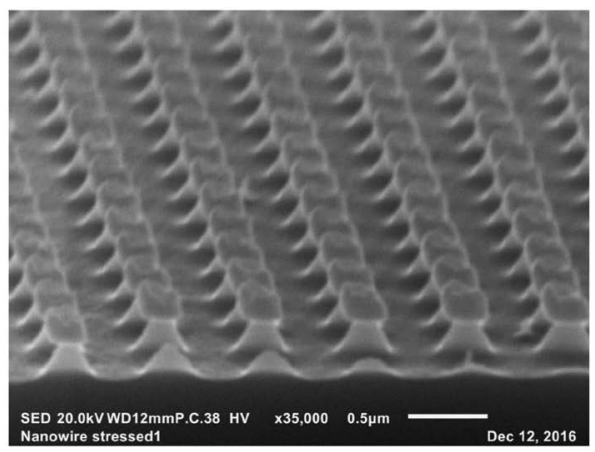

(a)

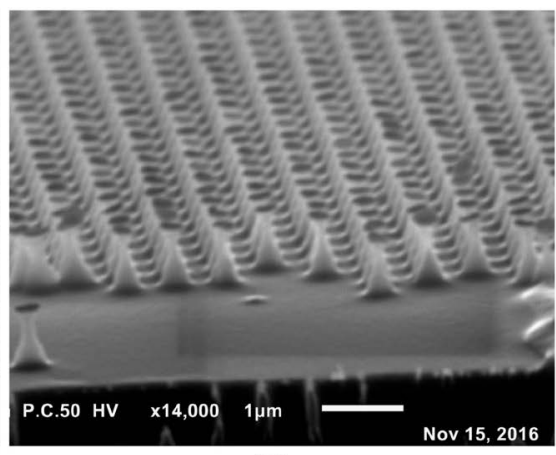

(b)

Figure 8. Both cases are etched for 25 minutes. Introducing Oxygen generates slanted side walls (a) Power set to 50 Watts. (b) Power set to 120 Watts. 


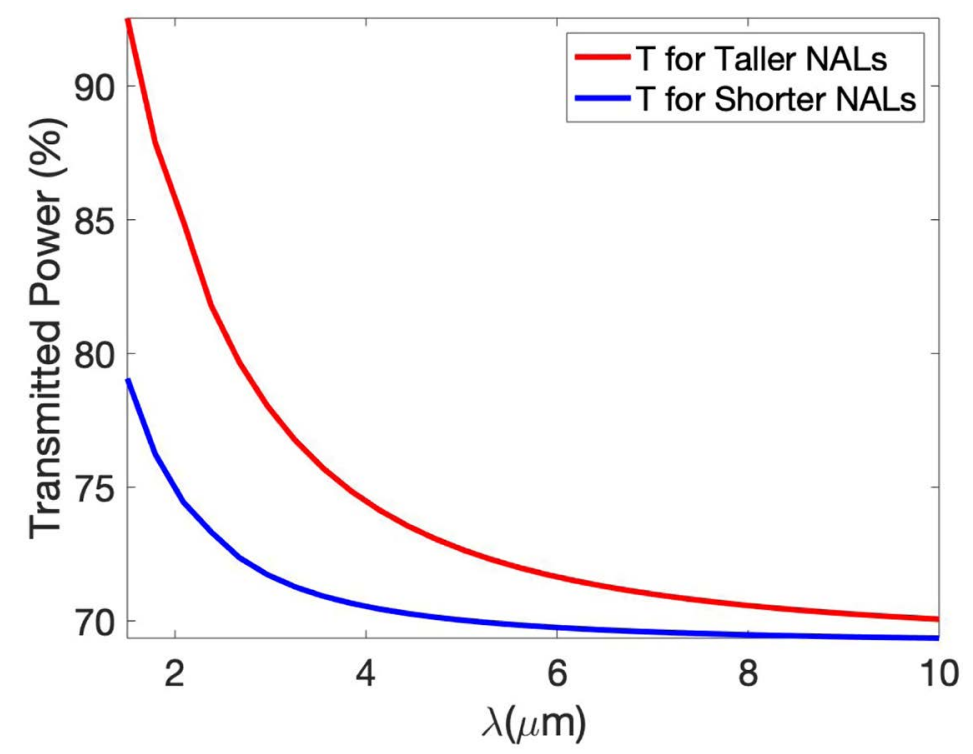

Figure 9. Transmission comparison for the tall and short NALs seen in Figure 8.

still have a low fill factor and one would need to come up with new solutions for lifting off metallic patterns that result in high fill factor at the bottom of the NALs is required for further improvement. While we do not investigate complex Maxwell equations, we should mention that the standing waves resulting from reflection of light from nanotextured substrate, and the E-field pattern of the light wave just above the substrate, is the reason behind the enhanced transmission of the light waves into bulk substrate. The E-field of the standing wave highly enhances just above substrate and distributes itself in such a way that the wave is least reflected on the border of space and substrate.

\section{Conclusion}

NALs etched out of a substrate have the same material as the substrate which minimizes thermal stresses. They are designed to optimize transmission of infrared light from air into silicon substrate. As long as the dimensions of the nanostructures are kept small compared to the wavelength of the light, the refractive index can be engineered in NALs through changing the "Fill-factor". Our investigation shows that reducing the height to $\frac{\lambda}{2 n_{\text {eff }}}$ still can result in above $99 \%$ transmission. This highly increases the mechanical stability of nanostructured surface. The results of RCWA show that we can gain a broadband transmission using the fabricated structures. The NALS profile allows a continuous transition of refractive index from air to the silicon substrate resulting in up to $99.1 \%$ transmission. Profiles close to the calculated results need to show that they are close to the structures generated on silicon using $\mathrm{CF}_{4}$ and $\mathrm{O}_{2}$ plasma in a single step, without changing the composition or plasma power during the etch. This highly reduces the complexity and cost of the etching process. The patterns are generated using IL. The periodicity of patterns generated by IL results in NALs capable of trans- 
mission without diffraction while minimizing the scattered light. This maintains the quality of transmitted infrared laser beams without any forward diffraction or any scattering common in random structures. The short height of the NALs (equivalent to a $\pi$ phase shift), would be big advantage since it retains the transmission peak above $99 \%$ while highly enhancing the mechanical stability.

\section{Acknowledgements}

Funding for this work was provided by the Center for High Technology Materials at the University of New Mexico.

\section{Conflicts of Interest}

The authors declare no conflicts of interest regarding the publication of this paper.

\section{References}

[1] Raut, H.K., Ganesh, V.A., Nair, A.S. and Ramakrishna, S. (2011) Anti-Reflective Coatings: A Critical, in-Depth Review. Energy \& Environmental Science, 4, 3779-3804. https://doi.org/10.1039/clee01297e

[2] Bouhafs, D., Moussi, A., Chikouche, A. and Ruiz, J. (1998) Design and Simulation of Antireflection Coating Systems for Optoelectronic Devices: Application to Silicon Solar Cells. Solar Energy Materials and Solar Cells, 52, 79-93. https://doi.org/10.1016/S0927-0248(97)00273-0

[3] Zhao, J., Wang, A., Altermatt, P. and Green, M. (1995) Twenty-Four Percent Efficient Silicon Solar Cells with Double Layer Antireflection Coatings and Reduced Resistance Loss. Applied Physics Letters, 66, 3636-3638. https://doi.org/10.1063/1.114124

[4] Askar, K., Phillips, B.M., Fang, Y., Choi, B., Gozubenli, N., Jiang, P. and Jiang, B. (2013) Self-Assembled Self-Cleaning Broadband Anti-Reflection Coatings. Colloids and Surfaces A: Physicochemical and Engineering Aspects, 439, 84-100. https://doi.org/10.1016/j.colsurfa.2013.03.004

[5] Xu, Y., Zhang, B., Fan, W.H., Wu, D. and Sun, Y.H. (2003) Sol-Gel Broadband Anti-Reflective Single-Layer Silica Films with High Laser Damage Threshold. Thin Solid Films, 440, 180-183. https://doi.org/10.1016/S0040-6090(03)00828-9

[6] Parizi, K.B., Peyvast, N., Mousavi, B.K., Mohajerzadeh, S. and Fathipour, M. (2010) Schottky Barrier Nano-MOSFET with an Asymmetrically Oxidized Source/Drain Structure. Solid-State Electronics, 54, 48-51. https://doi.org/10.1016/j.sse.2009.09.010

[7] Mousavi, B.K. (2018) Selected Applications of Silicon Nanopillar Arrays. Ph.D. Thesis, The University of New Mexico, Albuquerque, NM.

[8] Roy, A., Talarposhti, M.R., Normile, S.J., Zenyuk, I.V., De Andrade, V., Artyushkova, K., Serov, A. and Atanassov, P. (2018) Nickel-Copper Supported on a Carbon Black Hydrogen Oxidation Catalyst Integrated into an Anion-Exchange Membrane Fuel Cell. Sustainable Energy \& Fuels, 2, 2268-2275. https://doi.org/10.1039/C8SE00261D

[9] Liu, J., Talarposhti, M., Asset, T., Sabarirajan, D.C., Parkinson, D.Y., Atanassov, P. and Zenyuk, I.V. (2019) Understanding the Role of Interfaces for Water Management in PGM-free Electrodes in Polymer Electrolyte Fuel Cells. ACS Applied Ener 
gy Materials, 2, 3542-3553. https://doi.org/10.1021/acsaem.9b00292

[10] Mousavi, A.K., Abbas, K., Elahi, M.M.M., Lima, E., Moya, S., Butner, J.D., Pinon, D., Benga, A., Mousavi, B.K. and Leseman, Z.C. (2014) Pulsed Vacuum and Etching Systems: Theoretical Design Considerations for a Pulsed Vacuum System and Its Application to XeF 2 Etching of Si. Vacuum, 109, 216-222. https://doi.org/10.1016/j.vacuum.2014.07.028

[11] Mousavi, A., Kashamolla, M. and Leseman, Z. (2013) Improved Model for the Adhesion of $\mu$ Cantilevers: Theory and Experiments. Journal of Micromechanics and Microengineering, 23, Article ID: 115011. https://doi.org/10.1088/0960-1317/23/11/115011

[12] Kheyraddini Mousavi, A., Alaie, S. and Leseman, Z.C. (2016) Basic MEMS Actuators. In: Encyclopedia of Nanotechnology, Wiley, New York, 1-16. https://doi.org/10.1007/978-94-007-6178-0_292-2

[13] Mousavi, B.K., Mousavi, A.K., Hieber, T.J., Chen, J. and Leseman, Z.C. (2019) Mode II Adhesion Energy Analysis of Stiction-Failed Poly-Si Cantilevers Using a MEMS Load Cell. Journal of Micromechanics and Microengineering, 29, Article ID: 075013. https://doi.org/10.1088/1361-6439/ab173e

[14] Kheyraddini-Mousavi, A., Leseman, Z. and Kheyraddini-Mousavi, B. (2019) High Speed Michelson Interferometer Microscope. US Patent No. 10436570.

[15] Karbassian, F., Mousavi, B.K., Rajabali, S., Talei, R., Mohajerzadeh, S. and Asl-Soleimani, E. (2014) Formation of Luminescent Silicon Nanowires and Porous Silicon by Metal-Assisted Electroless Etching. Journal of Electronic Materials, 43, 1271-1279. https://doi.org/10.1007/s11664-014-3051-3

[16] Kheyraddini Mousavi, B., Behzadirad, M., Silani, Y., Karbasian, F., Kheyraddini Mousavi, A. and Mohajerzadeh, S. (2019) Metal-Assisted Chemical Etching of Silicon and Achieving Pore Sizes as Small as $30 \mathrm{~nm}$ by Altering Gold Thickness. Journal of Vacuum Science \& Technology A: Vacuum, Surfaces, and Films, 37, Article ID: 061402. https://doi.org/10.1116/1.5112776

[17] Kumar, A., Chaliyawala, H., Siddhanta, S. and Barshilia, H.C. (2016) Broadband Quasi-Omnidirectional Subwavelength Nanoporous Antireflecting Surfaces on Glass Substrate for Solar Energy Harvesting Applications. Solar Energy Materials and Solar Cells, 145, 432-439. https://doi.org/10.1016/j.solmat.2015.11.014

[18] Bett, A., Eisenlohr, J., Höhn, O., Bläsi, B., Benick, J., Repo, P., Savin, H., Goldschmidt, J. and Hermle, M. (2014) Front Side Antireflection Concepts for Silicon Solar Cells with Diffractive Rear Side Structures. 29 th European Photovoltaic Solar Energy Conference and Exhibition, Brussels, Belgium, 987-991. https://doi.org/10.1117/12.2051667

[19] Kang, G., Yoo, J., Ahn, J. and Kim, K. (2015) Transparent Dielectric Nanostructures for Efficient Light Management in Optoelectronic Applications. Nanotoday, 10, 22-47. https://doi.org/10.1016/j.nantod.2015.01.008

[20] Kim, J.J., Lee, Y., Kim, H.G., Choi, K.J., Kweon, H.S., Park, S. and Jeong, K.H. (2012) Biologically Inspired LED Lens from Cuticular Nanostructures of Firefly Lantern. Proceedings of the National Academy of Sciences of the United States of America, 109, 18674-18678. https://doi.org/10.1073/pnas.1213331109

[21] Schulze, M., Damm, M., Helgert, M., Kley, E.B., Nolte, S. and Tünnermann, A. (2012) Durability of Stochastic Antireflective Structures-Analyses on Damage Thresholds and Adsorbate Elimination. Optics Express, 20, 18348-18355. https://doi.org/10.1364/OE.20.018348

[22] Hobbs, D.S. and MacLeod, B.D. (2007) High Laser Damage Threshold Surface Re- 
lief Micro-Structures for Antireflection Applications. In: Laser-Induced Damage in Optical Materials. 2007, International Society for Optics and Photonics, Volume 6720, 67200L. https://doi.org/10.1117/12.754223

[23] Weng, B., Qiu, J., Yuan, Z., Larson, P.R., Strout, G.W. and Shi, Z. (2014) Responsivity Enhancement of Midinfrared PbSe Detectors Using CaF2 Nano-Structured Antireflective Coatings. Applied Physics Letters, 104, Article ID: 021109. https://doi.org/10.1063/1.4861186

[24] Hobbs, D.S. and MacLeod, B.D. (2005) Design, Fabrication, and Measured Performance of Anti-Reflecting Surface Textures in Infrared Transmitting Materials. In: Laser-Induced Damage in Optical Materials. 2005, International Society for Optics and Photonics, Orlando, FL, Volume 6720, 67200L. https://doi.org/10.1117/12.604532

[25] Kothary, P., Phillips, B.M., Leo, S.Y. and Jiang, P. (2016) Bioinspired Broadband Midwavelength Infrared Antireflection Coatings on Silicon. Journal of Vacuum Science \& Technology B, Nanotechnology and Microelectronics: Materials, Processing, Measurement, and Phenomena, 34, Article ID: 041807. https://doi.org/10.1116/1.4958794

[26] Zhou, L., Dong, X., Zhou, Y., Su, W., Chen, X., Zhu, Y. and Shen, S. (2015) Multiscale Micro-Nano Nested Structures: Engineered Surface Morphology for Efficient Light Escaping in Organic Light-Emitting Diodes. ACS Applied Materials \& Interfaces, 7, 26989-26998. https://doi.org/10.1021/acsami.5b08575

[27] Hobbs, D.S., MacLeod, B.D. and Sabatino, E. (2012) Continued Advancement of Laser Damage Resistant Optically Functional Microstructures. In: Laser-Induced Damage in Optical Materials. 2012, International Society for Optics and Photonics, Boulder, CO, Volume 8530, 85300O. https://doi.org/10.1117/12.976909

[28] Singh, V., Lin, P.T., Patel, N., Lin, H., Li, L., Zou, Y., Deng, F., Ni, C., Hu, J., Giammarco, J., et al. (2014) Mid-Infrared Materials and Devices on a Si Platform for Optical Sensing. Science and Technology of Advanced Materials, 15, Article ID: 014603. https://doi.org/10.1088/1468-6996/15/1/014603

[29] Du, Y., Liu, S., He, H., Jin, Y., Kong, F. and Guan, H. (2012) Laser-Induced Damage Properties of Antireflective Porous Glasses. Optics Communications, 285, 5512-5518. https://doi.org/10.1016/j.optcom.2012.07.120

[30] Thomas, I.M. (1986) High Laser Damage Threshold Porous Silica Antireflective Coating. Applied Optics, 25, 1481-1483. https://doi.org/10.1364/AO.25.001481

[31] Wang, Z., Zhang, R., Wang, S., Lu, M., Chen, X., Zheng, Y., Chen, L., Ye, Z., Wang, C. and Ho, K. (2015) Broadband Optical Absorption by Tunable Mie Resonances in Silicon Nanocone Arrays. Scientific Reports, 5, 7810. https://doi.org/10.1038/srep07810

[32] Mousavi, B.K., Mousavu, A.K., Busani, T., Zadeh, M.H. and Brueck, S. (2019) Nanostructured Anti-Reflection Coatings for Enhancing Transmission of Light. Journal of Applied Mathematics and Physics, 7, 3083-3100. https://doi.org/10.4236/jamp.2019.712217

[33] Mousavi, A.K., Atwater, M.A., Mousavi, B.K., Jalalpour, M., Taha, M.R. and Leseman, Z.C. (2014) Mechanical and Electrical Characterization of Entangled Networks of Carbon Nanofibers. Materials, 7, 4845-4853.

https://doi.org/10.3390/ma7064845 